\title{
The role of postprandial very-low-density lipoprotein in the development of atrial remodeling in metabolic syndrome
}

Hsiang-Chun Lee ${ }^{1,2,3,4,5^{*}}$ (D, Shyi-Jang Shin ${ }^{4}$, Jih-Kai Huang ${ }^{6}$, Ming-Yen Lin ${ }^{7}$, Yu-Hsun Lin ${ }^{3}$, Liang-Yin Ke ${ }^{1,2}$, He-Jiun Jiang ${ }^{8,9}$, Wei-Chung Tsai ${ }^{3,4}$, Min-Fang Chao ${ }^{10}$ and Yi-Hsiung Lin ${ }^{3}$

\begin{abstract}
Background: Negatively charged very-low-density lipoprotein (VLDL-X) in metabolic syndrome (MetS) patients exerts cytotoxic effects on endothelial cells and atrial myocytes. Atrial cardiomyopathy, manifested by atrial remodeling with a dilated diameter, contributes to atrial fibrillation pathogenesis and predicts atrial fibrillation development. The correlation of $\mathrm{VLDL}-\chi$ with atrial remodeling is unknown. This study investigated the association between VLDL- $X$ and remodeling of left atrium.
\end{abstract}

Methods: Consecutively, 87 MetS and 80 non-MetS individuals between 23 and 74 years old (50.6\% men) without overt cardiovascular diseases were included in the prospective cohort study. Blood samples were collected while fasting and postprandially (at $0.5,1,2$, and $4 \mathrm{~h}$ after a unified meal). VLDL was isolated by ultracentrifugation; the percentile concentration of VLDL-X (\%) was determined by ultra-performance liquid chromatography. The correlations of left atrium diameter ( $L A D)$ with variables including VLDL-X, LDL-C, HDL-C, triglycerides, glucose, and blood pressure, were analyzed by multiple linear regression models. A hierarchical linear model was conducted to test the independencies of each variable's correlation with LAD.

Results: The mean LAD was $3.4 \pm 0.5 \mathrm{~cm}$ in non-MetS subjects and $3.9 \pm 0.5 \mathrm{~cm}$ in MetS patients $(P<0.01)$. None of the fasting lipid profiles were associated with LAD. VLDL-X, BMI, waist circumference, hip circumference, and blood pressure were positively correlated with $\operatorname{LAD}($ all $P<0.05$ ) after adjustment for age and sex. Significant interactions between VLDL-X and blood pressure, waist circumference, and hip circumference were observed. When adjusted for obesity- and blood pressure-related variables, 2-h postprandial VLDL-X (mean $1.30 \pm 0.61 \%$ ) showed a positive correlation with LAD in MetS patients. Each 1\% VLDL-x increase was estimated to increase LAD by $0.23 \mathrm{~cm}$.

Conclusions: Postprandial VLDL- $X$ is associated with atrial remodeling particularly in the MetS group. VLDL-X is a novel biomarker and may be a therapeutic target for atrial cardiomyopathy in MetS patients.

Trial registration: ISRCTN 69295295. Retrospectively registered 9 June 2020.

Keywords: Atrial cardiomyopathy, Atrial fibrillation, Metabolic syndrome, Negatively charged, Postprandial, Verylow-density lipoprotein

\footnotetext{
* Correspondence: hclee@kmu.edu.tw

'Center for Lipid Biosciences, Kaohsiung Medical University Hospital, Kaohsiung, Taiwan

${ }^{2}$ Lipid Science and Aging Research Center, College of Medicine, Kaohsiung Medical University, Kaohsiung, Taiwan

Full list of author information is available at the end of the article
}

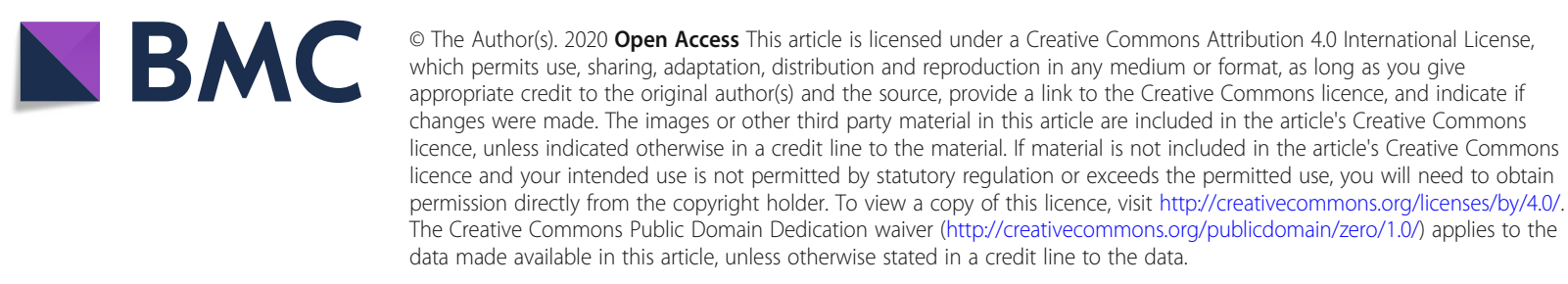




\section{Background}

Metabolic syndrome (MetS) is a major factor associated with the morbidity and mortality of cardiovascular diseases, including atrial fibrillation (AF) [1]. The association of MetS with AF has been identified in epidemiological studies [2-5]. Furthermore, some manifestations of MetS, such as hypertension, diabetes mellitus (DM), and obesity, are independently associated with AF and are attributed to pathogenic mechanisms of atrial remodeling and the development of AF [4-8]. Nevertheless, the findings from clinical studies of the correlation between dyslipidemia and $\mathrm{AF}$ are not consistent [9].

Elevated triglycerides are common in MetS and correlate with elevated cardiovascular risk [10]. Triglycerides are primarily carried by very low density lipoprotein (VLDL) in circulation [11]. In the postprandial state, the triglyceride level varies greatly and is affected largely by VLDL-related metabolism [12-14]; therefore, hypertriglyceridemia is preferentially defined by fasting triglyceride values [15]. Nevertheless, nonfasting triglyceride values have been shown to better predict cardiovascular disease risks $[16,17]$. The capacity to retain hemostasis upon food intake has been proposed to be important in normal human lipid and glucose metabolism [18]; a sequence of responses is elicited from the gastrointestinal system involving hormones and digestion enzymes, followed by nutritional absorption and delivery to organs and tissues. Abnormalities in postprandial lipid catabolism have been shown to be able to effectively detect early MetS [19].

Furthermore, VLDL remnants in the postprandial state have higher affinity for VLDL receptors [20]. VLDL receptors are expressed abundantly in the heart and are associated with hypertension-related ventricular hypertrophy [21]. Regarding atrial remodeling, we are the first group to examine VLDL-induced atrial remodeling and related mechanisms [9, 22-25]. Of note, the aforementioned changes can be induced only by VLDL in MetS patients but not VLDL in non-MetS subjects. On the basis of electrical charge (i.e., the abundance of negative charge), our colleagues developed a method for stratification of lipoproteins and found much higher cytotoxicity associated with VLDL in MetS patients with the highest abundance of negative charge $[23,26]$. Here, we tested the hypothesis that VLDL may be attributed to atrial remodeling, more so in the postprandial state than in the fasting state. We also hypothesized that negatively charged VLDL (referred to as VLDL- $X$ ) may be associated with atrial remodeling in MetS patients.

Left atrium (LA) diameter, a simple echocardiographic parameter, predicts AF recurrence risk after electrical cardioversion and is the first reported index of structural remodeling and a widely accepted predictive marker for AF [27]. On the other hand, noninvasive electrocardiography $\mathrm{P}$ wave indexes, such as the $\mathrm{P}$ wave duration, $\mathrm{PR}$ interval, and terminal force of $\mathrm{P}$ waves, have been reported as indicators of atrial electrical dysfunction [28-30]. The objectives of the current study were to determine the quantitative changes in lipids and glucose levels before and after meal intake and to evaluate the associations of VLDL- $\chi$ and markers of MetS with indexes of LA remodeling. The differences in postprandial variation in VLDL- $\chi$ between patients with and without LA remodeling were also evaluated.

\section{Methods}

\section{Study participant enrollment}

To determine the differences between MetS and nonMetS subjects, regarding of changes in lipids and glucose levels before and after meal intake, the associations by MetS of LA remodeling, and the correlation of VLDL- $X$ with LA remodeling, this prospective cohort study was conducted at a single Medical Center. Consecutively, 167 participants were enrolled (age range, 23-74 years old). The exclusion criteria included significant coronary heart disease, myocardial infarction, congenital heart diseases, heart failure, significant heart valve diseases, cerebrovascular diseases, cancers, insulin therapy, women with pregnancy or breastfeeding. The inclusion of MetS participants shall meet requirement of criteria with any 3 of the following components: (1) central obesity (waist $\geq 80 \mathrm{~cm}$ for women and $\geq 90 \mathrm{~cm}$ for men); (2) raised blood pressure (BP) (systolic BP $\geq 130 \mathrm{mmHg}$ or diastolic BP $\geq 85 \mathrm{mmHg}$ or treatment of previously diagnosed HTN); (2) raised fasting glucose $(\geq 100 \mathrm{mg} / \mathrm{dL}$ or diagnosed type $2 \mathrm{DM})$; (3) raised triglycerides $(\geq 150 \mathrm{mg} / \mathrm{dL}$ or on triglyceride-lowering treatment); and (4) reduced HDL-C $(<50 \mathrm{mg} / \mathrm{dL}$ for women and $<40 \mathrm{mg} / \mathrm{dL}$ for men). Among these participants, 87 and 80 had and did not have MetS, respectively. All study participants with MetS were seen by cardiologists or endocrinologists at Kaohsiung Medical University Hospital (Kaohsiung, Taiwan). The study protocol was approved by the Kaohsiung Medical University Hospital Institutional Review Board (IRB) (KMUHIRB-E(I)-20170256). An informed consent form was signed and returned by all participants before joining the study and undergoing plasma collection. This study adhered to the principles of the Declaration of Helsinki.

\section{Sample and demographic data collection}

All study participants were instructed to fast before beginning at midnight and came to the hospital at $8 \mathrm{AM}$. Each participant underwent two venous blood draws $\left(20 \mathrm{~mL}\right.$ for each sampling, in BD VACUETTE ${ }^{\oplus}$ EDTA Blood Tubes (Becton, Dickinson and Company, Franklin Lakes, NJ, USA)); one blood draw was performed in a fasting state, and the other was after finishing the combo meal of $620 \mathrm{cal}$ including a ham-egg sandwich, hashed 
browns, and a sweetened tea. To abide by the IRB approved protocol, in which the amount of blood drawing shall not exceed $40 \mathrm{~mL}$ over a week, a postprandial time was randomly selected for each participant from one of $0.5 \mathrm{~h}, 1 \mathrm{~h}, 2 \mathrm{~h}$, and $4 \mathrm{~h}$. In addition to blood sample collection, each participant underwent measurements of height, body weight, abdominal and hip circumferences, blood pressure and heart rate measurements. The medical record, if available, was reviewed, data related to medical history (hypertension and type $2 \mathrm{DM}$ ) and medication use were recorded.

\section{Laboratory testing for biochemical indicators and the quantification of negatively charged low-density lipoprotein (LDL-X) and negatively charged VLDL (VLDL-X)}

The analysis of biochemical parameters was performed in the Department of Laboratory Medicine at Kaohsiung Medical University Hospital according to the standard operating procedures. Technicians who performed tests were blinded to participants identity and data. Randomly duplicate samples were used to determine the analytical accuracy and measurement precision. As described previously, pairs of plasma samples (in fasting and postprandial states) were obtained from 167 participants. Plasma samples were immediately supplemented with the following after collection to prevent bacterial contamination and oxidation: protease inhibitor cocktail (Roche Diagnostics, Indianapolis, IN), $1 \%$ penicillin/streptomycin/neomycin mixture (Invitrogen, Carlsbad, CA), and 0.5 mM EDTA. Plasma LDL and VLDL were isolated by using sequential potassium bromide density-gradient ultracentrifugation between a density range of 1.006 and $1.063 \mathrm{~g} / \mathrm{mL}$. Fractions of LDL and VLDL samples that were isolated by density were resolved into subfractions with most negatively charged lipoproteins, i.e., LDL- $\chi$ and VLDL- $\chi$, respectively, by increasing the negative charge on UnoQ12 columns (BioRad, Hercules, CA) in the ion-exchange fastprotein liquid chromatography system (FPLC; GE Healthcare, Chicago, IL), as described previously [26, 31]. In short, the columns were first equilibrated with buffer $\mathrm{A}$ (0.02 M Tris-HCl, pH 8.0; 0.5 mM EDTA). With a multistep linear gradient of buffer $\mathrm{B}(1 \mathrm{M} \mathrm{NaCl}$ in buffer A) at a flow rate of $2 \mathrm{~mL} / \mathrm{min}$ under observation at $280 \mathrm{~nm}$, the LDL- $X$ and VLDL- $\chi$ subfractions were eluted, separately concentrated by using Centriprep filters (YM-30; EMD Millipore Corp., Billerica, MA) and sterilized by being passed through $0.22-\mu \mathrm{m}$ filters. The protein concentration of the LDL and VLDL samples was measured by using the Lowry method [31, 32].

\section{Electrocardiographic (ECG) parameters}

Twelve-lead ECG was performed by experienced medical technicians. The parameters that were measured and recorded by one experienced technician who was blinded to the other data and clinical information included $\mathrm{P}$ wave durations, PR intervals, QRS width, QTc intervals, and the terminal force of $\mathrm{P}$ waves in lead V1 [33]. Cardiac rhythms were interpreted, and any rhythm other than regular sinus rhythm (such as AF and/or flutter, pacemaker rhythm, ventricular tachycardia, supraventricular tachycardia, and second- or third-degree atrialventricular block) was discarded before further analyses.

\section{Echocardiographic assessment}

Echocardiography was performed by one experienced cardiologist using a transthoracic cardiac probe (Vivid 7; General Electric Medical Systems, Horten, Norway), with the participant in the left decubitus position. Twodimensional and two-dimensional-guided M-mode images were obtained, and LA diameter, left ventricle (LV) size, and LV function were assessed according to the standards of the American Society of Echocardiography [34]. LV ejection fraction (LVEF) was derived by the modified Simpson's method [34]. The raw data were measured and recorded while the researchers were blinded to the other data.

\section{Statistical analysis}

All continuous variables are presented as the mean \pm standard deviation. For all parameters examined in this study, the Shapiro-Wilk normality test was used to determine whether a random sample of values followed a normal distribution. To compare differences between the non-MetS and MetS groups, Student's t test was used for continuous data, and a chi-square test or Fisher's exact test was used for binary data. The associations of LA diameter and ECG parameters with body mass index (BMI), waist circumference, hip circumference, systemic blood pressure, pulse rate, fasting and postprandial plasma glucose, triglycerides, HDL-C, LDL- $X$ and VLDL- $\chi$ were evaluated by using the Pearson or Spearman (nonparametric) correlation. To evaluate the determining and confounding factors for LA diameter, simple linear regression and hierarchical multivariable linear regression with the stepwise method in the MetS group, non-MetS group, and total group were used to analyze all the variables, including age, hypertension, DM, BMI, waist circumference, hip circumference, heart rate, systolic blood pressure, diastolic blood pressure, VLDL- $\chi$, triglycerides, high-density lipoprotein cholesterol, LV size, LVEF, E/E' med, and E/E' lat. To validate robustness of main findings, several procedures were also carried out. First, a matching procedure by random selection of subjects from the non-MetS group by age within \pm 2 years was conducted in order to reduce imbalance of age distribution between two groups. Second, multivariable analyses were re-performed by MetS for the overall and the age-matched 47 pairs subjects. Results 
were considered statistically significant based on a $P$ value $<0.05$. Statistical analyses were performed by using the statistical package in GraphPad Prism (version 8; GraphPad Software, Inc., San Diego, CA, USA) software system and SPSS statistical software (version 22; IBM Corp., Armonk, NY, USA) and SAS 9.4 software (SAS Institute Inc., Cary, NC, USA).

\section{Results}

\section{Subject characteristics}

Among 80 non-MetS and 87 MetS subjects participating in this study, sex was matched between groups (Table 1). The MetS group was older than the non-MetS group (52.7 \pm 11.5 years vs. $44.4 \pm 10.2$ years, $P<0.0001$ ). Among 87 MetS subjects, 83.9 and $78.2 \%$ had hypertension and DM, respectively. Markers of obesity, including BMI, waist circumference, and hip circumference, were greater in the MetS group. Similarly, blood pressure and heart rate were higher in the MetS group. In regard to biochemistry data, plasma hemoglobin A1c and fasting glucose were higher in the MetS group. While creatinine was similar in both groups, aspartate aminotransferase (AST) and alanine aminotransferase (ALT) were higher in the MetS group. In regard to lipid profiles, while the MetS group had higher fasting triglycerides and HDL-C, total cholesterol and LDL-C were similar in both groups. In addition, the MetS group had a higher plasma level of uric acid.

\section{Changes in glucose and triglycerides from fasting to after} the unified meal in the MetS and non-MetS groups

Glucose and triglycerides were higher in the MetS group under fasting conditions, while glucose levels decreased $4 \mathrm{~h}$ after the meal, and triglyceride levels were still elevated in the MetS group (Fig. 1a and b).

\section{Changes in HDL-C, LDL-C, LDL- $\chi$ and VLDL- $\chi$ from fasting to after the unified meal in the MetS and non-MetS groups}

HDL-C was lower in the MetS group at fasting and exhibited a decreasing trend after the meal at $2 \mathrm{~h}$ and $4 \mathrm{~h}$ (Fig. 1c), whereas the postprandial decrease in HDL-C was not present in the non-MetS group. Unlike the results for HDL-C, the plasma LDL-C at fasting and the change after a meal were similar in both groups (Fig. 1d). While LDL-C did not change after a meal, the negatively charged LDL and LDL- $\chi$ in the MetS group decreased $4 \mathrm{~h}$ after the meal (Fig. 1e). The decrease at $4 \mathrm{~h}$ in LDL- $\chi$ did not occur in the non-MetS group. At fasting (0 h, Fig. 1f), the negatively charged VLDL- $\chi$ was similar in both groups but was significantly higher in the MetS group than in the

Table 1 Demographics of the study population

\begin{tabular}{|c|c|c|c|}
\hline Variables & Non-MetS $(n=80)$ & MetS $(n=87)$ & $P$ value \\
\hline Age, years & $44.4 \pm 10.2$ & $52.7 \pm 11.5$ & $<0.0001$ \\
\hline Male, n (\%) & $39(48.1)$ & $46(52.9)$ & 0.4477 \\
\hline Hypertension, n (\%) & $0(0)$ & $73(83.9)$ & - \\
\hline Diabetes mellitus, n (\%) & $5(6.2)$ & $68(78.2)$ & $<0.0001$ \\
\hline $\mathrm{BMI}, \mathrm{kg} / \mathrm{m}^{2}$ & $22.3 \pm 3.0$ & $29.2 \pm 4.7$ & $<0.0001$ \\
\hline Waist circumference, $\mathrm{cm}$ & $75.7 \pm 8.2$ & $97.6 \pm 9.7$ & $<0.0001$ \\
\hline Hip circumference, $\mathrm{cm}$ & $94.3 \pm 6.9$ & $104.0 \pm 9.2$ & $<0.0001$ \\
\hline Systolic BP, mmHg & $116.0 \pm 9.0$ & $143.5 \pm 21.3$ & $<0.0001$ \\
\hline Diastolic BP, mmHg & $70.1 \pm 7.3$ & $85.9 \pm 15.1$ & $<0.0001$ \\
\hline Heart rate, beats/min & $75.3 \pm 10.0$ & $84.2 \pm 12.8$ & $<0.0001$ \\
\hline \multicolumn{4}{|l|}{ Biochemistry } \\
\hline $\mathrm{HbA} 1 \mathrm{c}, \%$ & $5.4 \pm 0.2$ & $6.7 \pm 0.9$ & $<0.0001$ \\
\hline AST, IU/L & $17.7 \pm 7.1$ & $24.8 \pm 8.9$ & $<0.0001$ \\
\hline ALT, IU/L & $20.4 \pm 5.4$ & $32.0 \pm 18.4$ & $<0.0001$ \\
\hline Creatinine, mg/dL & $0.7 \pm 0.2$ & $0.8 \pm 0.2$ & 0.3018 \\
\hline Glucose, mg/dL & $86.1 \pm 9.5$ & $119.8 \pm 26.4$ & $<0.0001$ \\
\hline Total cholesterol, mg/dL & $187.9 \pm 35.0$ & $187.6 \pm 30.7$ & 0.9598 \\
\hline $\mathrm{HDL}-\mathrm{C}, \mathrm{mg} / \mathrm{dL}$ & $67.7 \pm 14.4$ & $41.5 \pm 9.0$ & $<0.0001$ \\
\hline $\mathrm{LDL}-\mathrm{C}, \mathrm{mg} / \mathrm{dL}$ & $116.5 \pm 30.9$ & $124.7 \pm 27.6$ & 0.1258 \\
\hline Triglycerides, mg/dL & $85.6 \pm 27.4$ & $208.7 \pm 100.3$ & $<0.0001$ \\
\hline Uric acid, mg/dL & $4.9 \pm 1.1$ & $5.8 \pm 1.3$ & $<0.0001$ \\
\hline
\end{tabular}




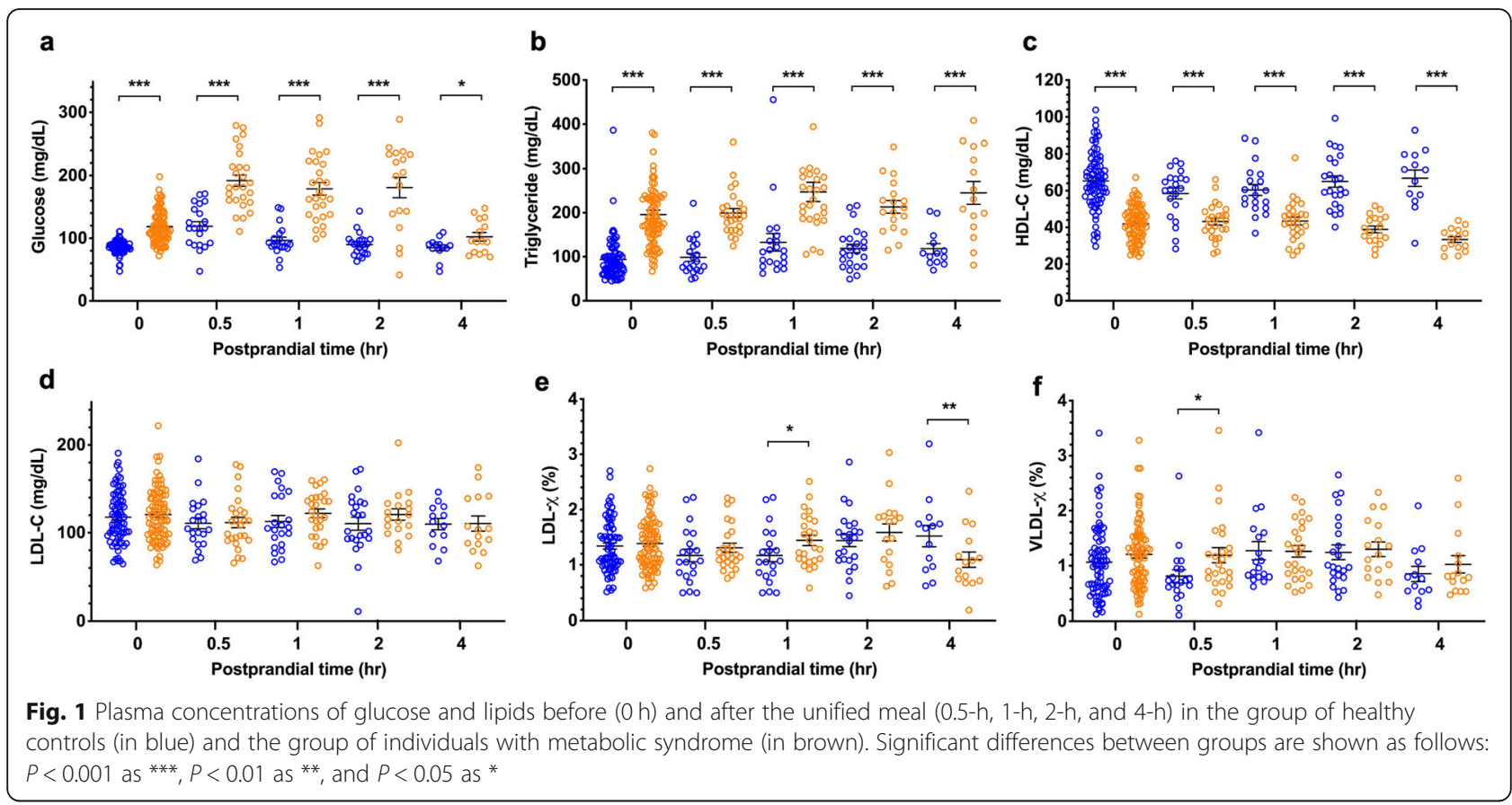

non-MetS group $0.5 \mathrm{~h}$ after the meal. VLDL- $\chi$ decreased $4 \mathrm{~h}$ after the meal in both groups.

\section{LA dilation as a marker for atrial structural remodeling} Among 167 non-MetS and MetS subjects participating in this study, subjects of age between 40 and 60 years were recruited to undergo 12-lead ECG and standard echocardiography. The characteristics of 62 non-MetS and 68 MetS subjects who had completed the heart examinations are shown in Table 2. Similar to the overall subjects, the MetS group was more obese, had higher blood pressure, and had a higher heart rate than the non-MetS group. Regarding ECG parameters, the PR interval was wider in the MetS group than in the nonMetS group $(167.6 \pm 20.0 \mathrm{msec}$ vs. $156.2 \pm 24.9 \mathrm{msec}, P=$ 0.0064), whereas the $P$ wave duration, terminal force of $\mathrm{P}$ waves, QRS width and QTc interval were similar in both groups. Regarding echocardiography, LV function was similar in both groups (LVEF, MetS $67.3 \pm 7.1 \%$ vs. non-MetS $63.9 \pm 11.3 \%, P=0.0515)$, whereas the diameter of the aorta, LA and size of LV were larger in the MetS group (Table 2). The ratios of mitral peak velocity of early filling (E) to early diastolic mitral annular velocity (E') as markers for diastolic function and indicators for LV filling pressure were higher in the MetS group (Table 2). The aforementioned results showed evidence of LA and LV remodeling in MetS participants in this study, and this remodeling was associated with diastolic dysfunction of the LV without overt systolic dysfunction.

\section{LA dilation was associated with central obesity and hypertension}

The LA diameter was shown to significantly correlate with BMI and waist and hip circumferences in both the non-MetS (Fig. 2a-c) and MetS (Fig. 2d-f) groups. The LA diameter was also positively correlated with blood pressure (Fig. 3) and with diastolic blood pressure in the MetS group (Fig. 3e). There was no significant correlation to LA diameter as for either plasma glucose (Suppl. Figure 1), triglyceride (Suppl. Figure 2), VLDL-cholesterol (Suppl. Figure 3), or LDL- $\chi$ (Suppl. Figure 4).

\section{Correlation of LA size with fasting and postprandial VLDL-X}

The results of the analysis of the correlation between LA diameter and fasting and $0.5-\mathrm{h}, 1-\mathrm{h}, 2-\mathrm{h}$, and $4-\mathrm{h}$ postprandial VLDL- $X$ are shown in Fig. 4. In the MetS group, the LA size was significantly correlated with fasting $(P=$ 0.014; Fig. 4f) and 2-h postprandial VLDL- $\chi(P=0.004$; Fig. 4i). Similarly, the LA size was also significantly correlated with 2-h postprandial VLDL-X in the non-MetS group $(P=0.033$; Fig. $4 \mathrm{~d})$.

\section{Two-hour postprandial VLDL-X is correlated with $\mathrm{P}$ wave duration}

Markers of electrical remodeling on $\mathrm{P}$ waves were measured and analyzed (Fig. 5a-f). The P wave duration was significantly correlated with 2-h postprandial VLDL$X$ in the MetS group $(P=0.036$; Fig. $5 \mathrm{~d})$, and the PR interval tended to be correlated with VLDL- $\chi$ (Fig. 5e) 
Table 2 Characteristics of subjects included in the echocardiographic study

\begin{tabular}{|c|c|c|c|}
\hline Variables & Non-MetS $(n=62)$ & MetS $(n=68)$ & $P$ value \\
\hline Age, years & $48.7 \pm 7.0$ & $51.9 \pm 9.4$ & 0.0351 \\
\hline Male, n (\%) & $31(50.0)$ & $37(54.4)$ & 0.4669 \\
\hline Hypertension, n (\%) & $0(0)$ & $58(85.3)$ & - \\
\hline Diabetes mellitus, n (\%) & $5(8.1)$ & $49(72.1)$ & $<0.0001$ \\
\hline $\mathrm{BMI}, \mathrm{kg} / \mathrm{m}^{2}$ & $22.7 \pm 3.0$ & $29.0 \pm 3.8$ & $<0.0001$ \\
\hline Waist circumference, cm & $78.2 \pm 8.5$ & $97.8 \pm 9.7$ & $<0.0001$ \\
\hline Hip circumference, $\mathrm{cm}$ & $95.2 \pm 6.5$ & $103.9 \pm 8.6$ & $<0.0001$ \\
\hline Systolic BP, mmHg & $116.4 \pm 10.3$ & $142.8 \pm 20.7$ & $<0.0001$ \\
\hline Diastolic BP, mmHg & $71.2 \pm 7.9$ & $87.2 \pm 14.4$ & $<0.0001$ \\
\hline Heart rate, beats/min & $73.9 \pm 9.2$ & $85.0 \pm 13.8$ & $<0.0001$ \\
\hline \multicolumn{4}{|l|}{ Electrocardiogram Parameters } \\
\hline $\mathrm{P}, \mathrm{msec}$ & $105.6 \pm 14.2$ & $108.9 \pm 13.2$ & 0.1872 \\
\hline $\mathrm{PR}$, msec & $156.2 \pm 24.9$ & $167.6 \pm 20.0$ & 0.0064 \\
\hline Terminal force of $\mathrm{P}, \mathrm{mVS}$ & $1.6 \pm 1.5$ & $1.7 \pm 1.6$ & 0.8157 \\
\hline QRS, msec & $85.7 \pm 10.8$ & $88.5 \pm 16.5$ & 0.2735 \\
\hline QTc, msec & $405.0 \pm 21.5$ & $405.5 \pm 37.8$ & 0.9292 \\
\hline \multicolumn{4}{|l|}{ Echocardiographic Parameters } \\
\hline Aorta diameter, cm & $3.2 \pm 0.3$ & $3.4 \pm 0.4$ & 0.0012 \\
\hline LA diameter, cm & $3.4 \pm 0.5$ & $3.9 \pm 0.5$ & $<0.0001$ \\
\hline LA volume, mL & $48.9 \pm 15.4$ & $70.6 \pm 18.2$ & $<0.0001$ \\
\hline LVEDD, cm & $4.6 \pm 0.5$ & $4.8 \pm 0.5$ & 0.0049 \\
\hline LVEDV, mL & $95.9 \pm 30.4$ & $126.0 \pm 37.2$ & $<0.0001$ \\
\hline LV mass, gram & $107.7 \pm 23.7$ & $133.7 \pm 28.8$ & $<0.0001$ \\
\hline$E F, \%$ & $63.9 \pm 11.3$ & $67.3 \pm 7.1$ & 0.0515 \\
\hline$E / E^{\prime}$ (med) & $7.7 \pm 2.3$ & $9.8 \pm 2.7$ & $<0.0001$ \\
\hline E/E' (lat) & $5.8 \pm 2.2$ & $6.7 \pm 1.8$ & 0.0169 \\
\hline Valve disease, n (\%) & 0 & 0 & - \\
\hline
\end{tabular}

BMI Body mass index, BP Blood pressure, LA Left atrium, LVEDD Left ventricle end-diastolic dimension, LVEDV Left ventricle end-diastolic volume, LVEF Left ventricle ejection fraction, $E / E^{\prime}(m e d)$ The ratio of mitral peak velocity of early filling $(E)$ to early diastolic mitral annular velocity $\left(E^{\prime}\right)$ at medial mitral ring, $E / E^{\prime}$ (lat) The ratio of mitral peak velocity of early filling (E) to early diastolic mitral annular velocity ( $\left.E^{\prime}\right)$ at the lateral mitral ring

a

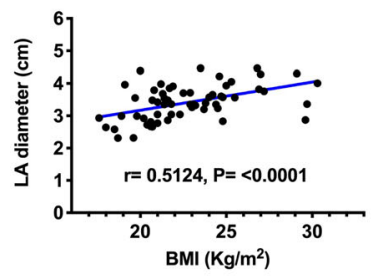

d

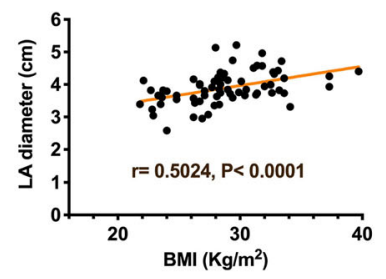

b

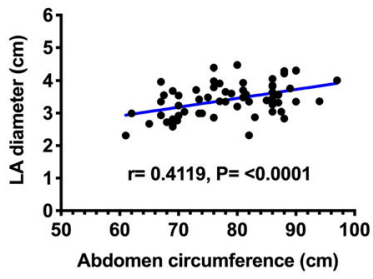

e

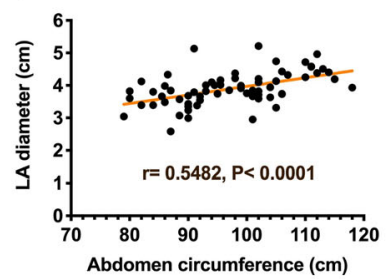

c

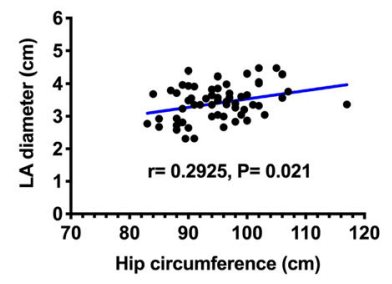

f

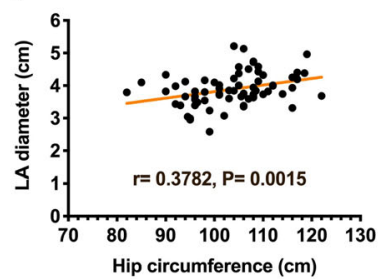

Fig. 2 Correlation between left atrial (LA) diameter and the parameters of body size and central obesity body mass index and waist and hip circumferences in individuals without (in blue, $\mathbf{a}-\mathbf{c}$ ) and with metabolic syndrome (in brown, $\mathbf{d}-\mathbf{f}$ ). Each correlation factor and $P$ value are labeled 


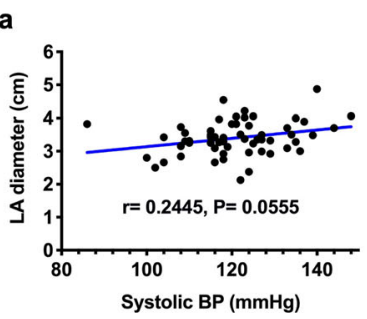

d

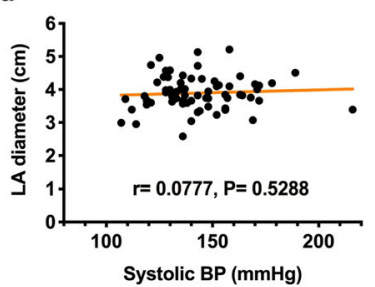

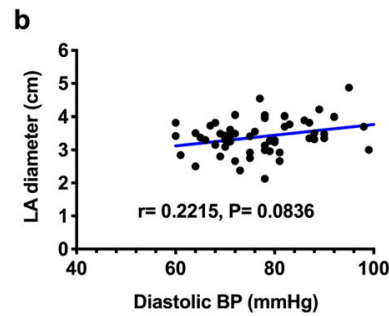
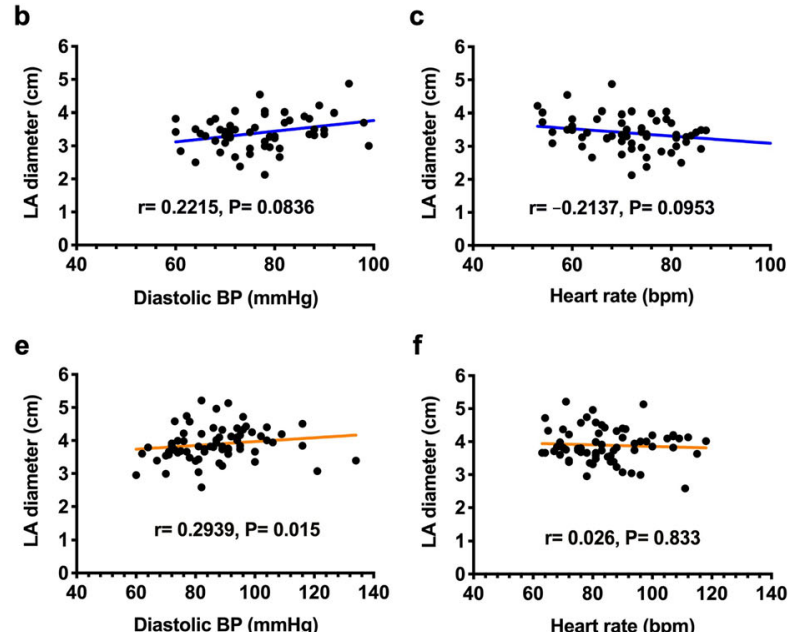

f

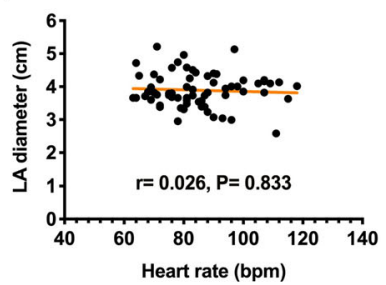

Fig. 3 Correlation between left atrial (LA) diameter and systolic and diastolic blood pressure (BP) and heart rate in individuals without (in blue, ac) and with metabolic syndrome (in brown, $\mathbf{d}$-f). Each correlation factor and $P$ value are labeled

but without a significant correlation in the non-MetS group (Fig. 5a-c).

\section{Different kinetics of VLDL-X in MetS subjects with larger LA size}

The changes in VLDL- $\chi$ from fasting to $2 \mathrm{~h}$ after the meal are shown in Fig. 6. The pattern of changes was different between the non-MetS and MetS groups $(P=0.0001$; Fig. 6a). In the MetS group with dilated LA (diameter $\geqq 3.8 \mathrm{~cm}$ ), VLDL- $\chi$ increased after the meal compared with the level in the fasting state, and VLDL- $X$ remaining steady in those with LA diameters smaller than $3.8 \mathrm{~cm}(P<0.0001$; Fig. $6 \mathrm{~b})$.
VLDL- $\chi$ was independently correlated with LA diameter in the MetS group

There was a significant correlation between VLDL- $X$ and blood pressure and waist and hip circumference. After adjusting for variables of obesity and blood pressure, VLDL- $\chi$ showed an independent positive correlation with LA diameter in the MetS group (Table 3). Each 1\% increase in VLDL- $X$ was estimated to increase the LA diameter by $0.23 \mathrm{~cm}(P=0.028)$. The other independent determining factor of LA diameter is waist circumference.

For all subjects in this study, the univariate analysis revealed four factors that were significantly correlated with LA diameter, including waist circumference, LVED D, LV end-diastolic volume (LVEDV), and E/E' med.

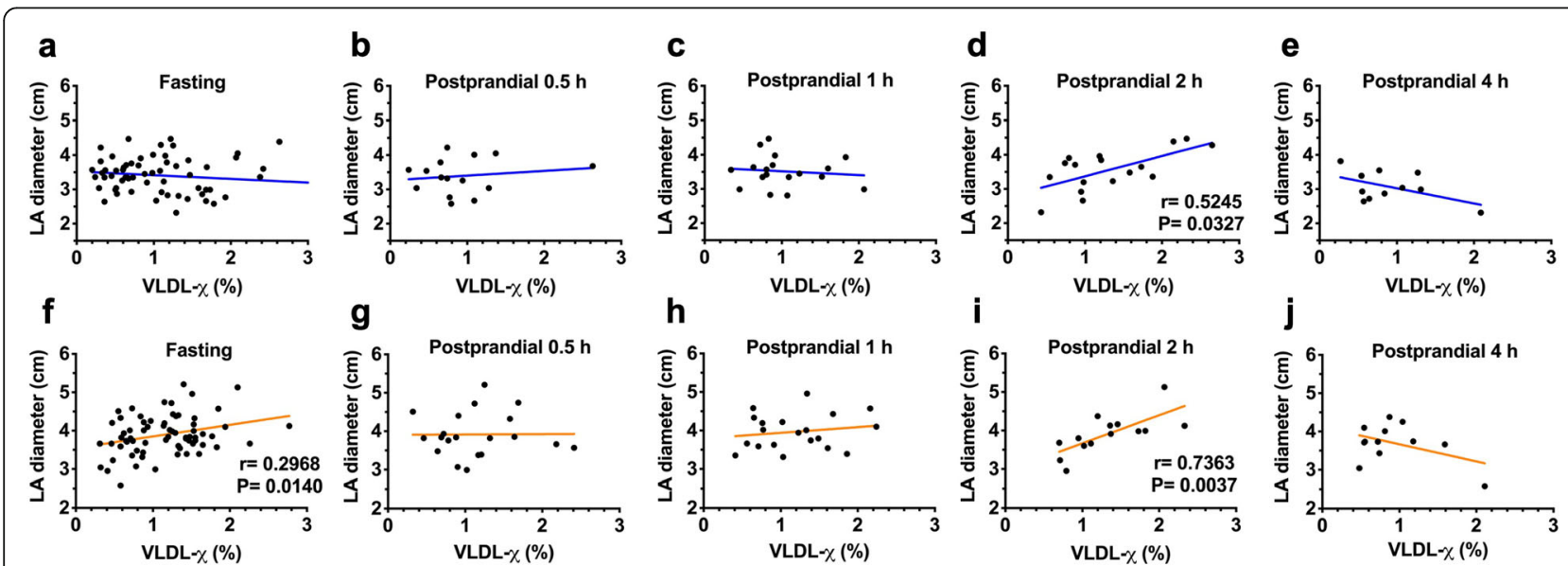

Fig. 4 Correlation between left atrial (LA) diameter and negatively charged very-low-density lipoprotein as a percentage (VLDL- $\chi$ ) before (fasting) and after breakfast (postprandial 0.5-h, 1-h, 2-h, and 4-h) in individuals without (in blue, a-e) and with metabolic syndrome (in brown, $\mathbf{f - j}$ ). A significant correlation (with $P<0.05$ ) is indicated with each correlation factor and $P$ value 


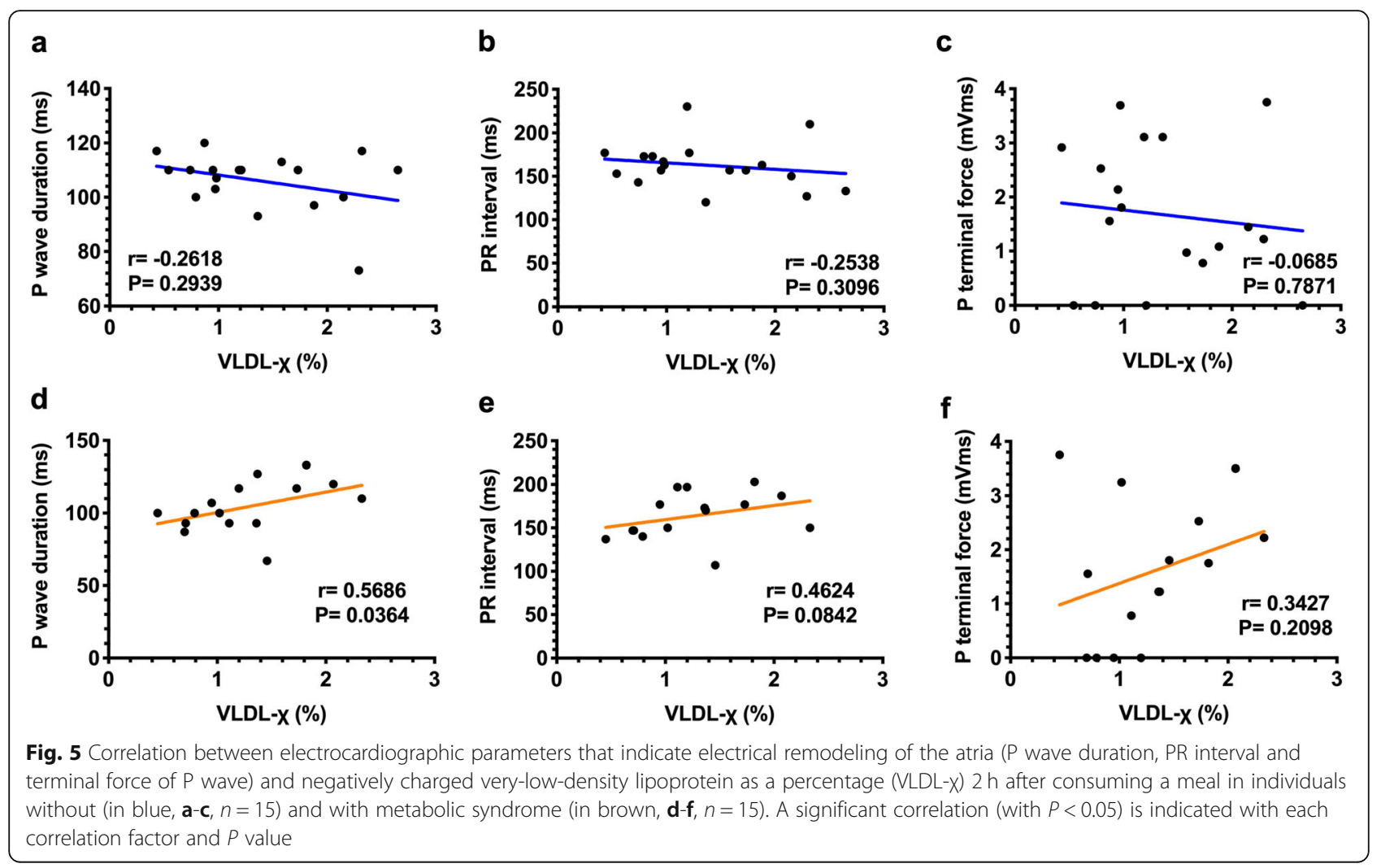

Among those factors, waist circumference and LVEDD remained independent determinants of LA diameter in multivariable analysis (see Suppl. Table S1). Among non-MetS subjects, age, BMI, and LVEDD were significantly and independently correlated with LA diameter (see Suppl. Tables S2-S3). Similar findings were found for the MetS group after matching our study subjects by age (Suppl. Tables S1 and S3).

\section{Discussion}

The main results from this study are as follows: (1) the postprandial kinetics of glucose, triglycerides, HDL-C,
LDL-C, LDL- $\chi$ and VLDL- $\chi$ were determined; (2) LA dilation was associated with markers of central obesity, hypertension, and postprandial VLDL- $\chi$; (3) 2-h postprandial VLDL- $X$ was associated with $P$ wave duration in electrocardiography and independently correlated with LA diameter; and (4) MetS subjects whose VLDL-X increased after the meal had a larger LA diameter than those whose VLDL- $\chi$ decreased.

The metabolism of VLDL is an important determinant of LDL formation and triglyceride levels. Both normal and hypertriglyceridemic VLDL molecules contain apoB100 and apoE. Hypertriglyceridemic VLDL has a higher
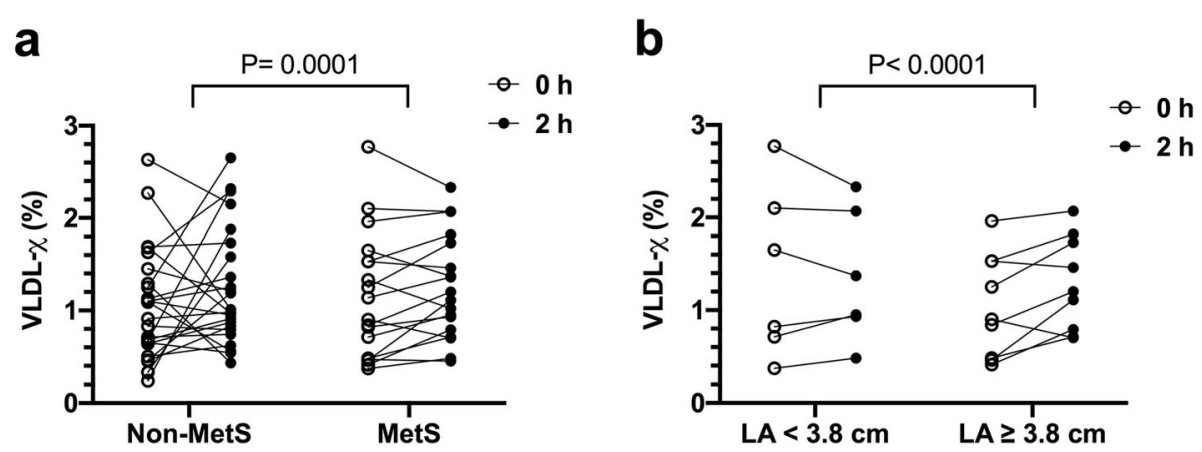

Fig. $\mathbf{6}$ a The change in plasma VLDL-x from fasting $(0 \mathrm{~h})$ to $2 \mathrm{~h}$ after breakfast in the nonmetabolic syndrome (non-MetS) group was different from that in the MetS group. $\mathbf{b}$ In the MetS group, subjects with an enlarged left atrial diameter $(\geq 3.8 \mathrm{~cm})$ had exhibited increases in VLDL-X postprandially 
Table 3 Univariate and multivariable linear regression of determining factors of LA diameter in MetS group

\begin{tabular}{|c|c|c|c|c|c|c|c|}
\hline & \multicolumn{3}{|c|}{ Univariate linear regression } & \multicolumn{4}{|c|}{ Multivariable linear regression $^{a}$} \\
\hline & $\begin{array}{l}\text { Regression } \\
\text { coefficient }\end{array}$ & $\begin{array}{l}95 \% \text { confidence } \\
\text { interval }\end{array}$ & $p$ value & $\begin{array}{l}\text { Regression } \\
\text { coefficient }\end{array}$ & $\begin{array}{l}95 \% \text { confidence } \\
\text { interval }\end{array}$ & $\mathbf{T}$ & $p$ value \\
\hline$\overline{\text { Age }}$ & -0.003 & $(-0.018,0.012)$ & 0.684 & & & & \\
\hline Hypertension & 0.480 & $(0.298,0.661)$ & $<0.001^{*}$ & & & & \\
\hline Diabetes mellitus & 0.330 & $(0.137,0.523)$ & $0.001^{*}$ & & & & \\
\hline $\mathrm{BMl}$ & 0.053 & $(0.02,0.087)$ & $0.02^{*}$ & & & & \\
\hline Waist circumference & 0.027 & $(0.015,0.039)$ & $<0.001^{*}$ & 0.028 & $(0.015,0.040)$ & 4.544 & $<0.001^{*}$ \\
\hline Hip circumference & 0.02 & $(0.004,0.035)$ & $0.012^{*}$ & & & & \\
\hline Systolic BP & -0.002 & $(-0.008,0.005)$ & 0.646 & & & & \\
\hline Diastolic BP & 0.000 & $(-0.009,0.01)$ & 0.949 & & & & \\
\hline Heart rate & -0.005 & $(-0.015,0.005)$ & 0.34 & & & & \\
\hline Triglycerides & $<0.001$ & $(-0.001,0.002)$ & 0.662 & & & & \\
\hline $\mathrm{HDL}-\mathrm{C}$ & -0.009 & $(-0.024,0.005)$ & 0.187 & & & & \\
\hline VLDL-X & 0.258 & $(0.034,0.482)$ & $0.024^{*}$ & 0.225 & $(0.025,0.425)$ & 2.25 & $0.028^{*}$ \\
\hline LVEDD & 0.166 & $(-0.52,0.385)$ & 0.134 & & & & \\
\hline LVEDV & 0.005 & $(0.002,0.009)$ & $0.005^{*}$ & & & & \\
\hline $\mathrm{EF}$ & 0.018 & $(-0.00,0.037)$ & 0.067 & & & & \\
\hline E/E' med & -0.015 & $(-0.067,0.037)$ & 0.567 & & & & \\
\hline \multirow[t]{3}{*}{ E/E' lat } & -0.008 & $(-0.085,0.068)$ & 0.828 & & & & \\
\hline & & & & \multicolumn{4}{|c|}{ Model adjusted R-square } \\
\hline & & & & 0.37 & & & \\
\hline
\end{tabular}

${ }^{a}$ Hierarchical multivariable linear regression in stepwise method with variables including age, hypertension, diabetes mellitus, body mass index (BMI), waist circumference, hip circumference, systolic blood pressure (BP), diastolic BP, heart rate, triglycerides, very-low-density lipoprotein (VLDL)-X, high-density lipoprotein cholesterol (HDL-C), left ventricle end-diastolic dimension (LVEDD), left ventricle end-diastolic volume (LVEDV), ejection fraction (EF), ratio of mitral flow E velocity and tissue Doppler $E^{\prime}$ velocity of the medical and lateral mitral ring, i.e., $E / E^{\prime}$ med and $E / E^{\prime}$ lat

content of apoE, which facilitates its affinity to VLDL receptors [35]. The tissue distribution of VLDL receptors is quite different from that of LDL receptors. The VLDL receptor is abundant in tissue largely utilizing fatty acids as an energy source, including heart, muscle, and adipose tissue, and it mediates cellular catabolism and regulates the levels of lipoprotein lipase [36, 37]. Whether the modulation of VLDL receptor expression is involved in VLDL-X-related LA remodeling needs further study.

The importance of postprandial hyperlipidemia in the context of atherosclerosis was suggested early in 1979 [38] and was later increasingly explored in recent years $[10,16,17]$. In 2016, experts reached a consensus regarding the complementary value of nonfasting measurements of lipid profiles for clinical and laboratory medicine [39]. More recently, Nakajima et al. reported evidence to disclose the predictive role of remnant lipoproteins in coronary heart diseases [20]. They also reported that compared to fasting VLDL, postprandial VLDL has a higher affinity for VLDL receptors and easier internalization [40]. Accordingly, it might be suggested that the postprandial VLDL- $X$ has larger internalization and results in cytotoxicity in atrial tissue and ultimately significant remodeling of the atrium. To the best of our knowledge, our study is the first to report the correlation of postprandial VLDL- $X$ with LA remodeling.

The association of markers of central obesity with LA size is consistent with results derived by other clinical studies [41, 42]. Obesity, as a well-identified risk factor for AF, causes discrete atrial cardiomyopathy in animal models [43, 44]. Experimental hypertensive rats have fibrosis, inflammation, atrial wavelength shortening and calcium current changes in the LA [45]. Patients with obesity often have coexisting hypertension and dyslipidemia; therefore, it is difficult to identify the major cause of atrial remodeling. In this study, the postprandial VLDL- $\chi$ was shown as an independent determinant for LA dilatation.

\section{Study strength and limitations}

This study examined the role of VLDL- $\chi$ not only for fasting state but also for postprandial state with blood sampling at different time points related to the unified meal. By the multiple-sampling methods, this study was therefore able to unveil the significant role for VLDL- $\chi$ in 
structural and electrical remodeling of LA, which results in vulnerability to AF. This study also determined the specific time point as of postprandial $2 \mathrm{~h}$, which is coincident with the peak phase for VLDLs on transportation of lipids in circulation after food intake. Here, there are several study limitations to be indicated. First, obtaining postprandial samples in a random manner reduced postprandial data size, and may introduce bias in the results. Second, the VLDL- $\chi$ was not determined as of real concentrations. Third, the age between two groups with and without MetS were not matched. This limitation had been overcome by using the age-matching procedure of statistics. Similar multivariable analysis results were found in the matched groups (MetS group: $49.9 \pm 7.3$ vs. non-MetS group: $49.7 \pm 7.2, P=0.89$ ) (in Suppl. Table S1 to S3). Lastly, the impact of age on postprandial VLDL- $\chi$ could not be analyzed with relatively small sample size in this study.

\section{Conclusions}

A significant correlation between postprandial plasma VLDL- $\chi$ and LA remodeling, particularly in the MetS group, was derived by this study. Despite other factors, such as hypertension and obesity, which also cause LA remodeling in MetS patients, plasma VLDL- $X$ is still attributed to LA remodeling. We suggest that the metabolism of VLDL and the characteristics of postprandial VLDL- $\chi$ are the major determinants of the progression of LA remodeling, particularly in MetS patients. The pathogenesis of AF is complex and can involve a combination of etiological factors and metabolism-associated elements. In many patients, AF is not curable at the time of diagnosis due to long-lasting and overt atrial remodeling with extensive fibrosis. For MetS patients, the association of postprandial plasma VLDL-X on LA remodeling may give some insights for early prevention of AF.

\section{Supplementary information}

Supplementary information accompanies this paper at https://doi.org/10. 1186/s12944-020-01386-5.

Additional file 1: Table S1. Univariate and multivariable linear regression of determining factors of LA diameter in the matched groups $(n=94)$. Table S2. Multivariable linear regression of determining factors of LA diameter by metabolic syndrome (MetS). Table S3. Multivariable linear regression of determining factors of LA diameter by metabolic syndrome (MetS) in the matched groups $(n=94)$. Figure S1. Correlation between left atrial (LA) diameter and plasma concentration of glucose before (fasting) and after the unified meal (postprandial 0.5, 1, 2, and $4 \mathrm{~h}$ ) in participants without MetS (in blue, A-E) and with MetS (in orange, F-J). Figure S2. Correlation between left atrial (LA) diameter and plasma concentration of triglyceride before (fasting) and after the unified meal (postprandial $0.5,1,2$, and $4 \mathrm{~h}$ ) in participants without MetS (in blue, A-E) and with MetS (in orange, F-J). Figure S3. Correlation between left atrial (LA) diameter and plasma concentration of very-low-density lipoprotein cholesterol (VLDL-C) before (fasting) and after the unified meal (postprandial $0.5,1,2$, and $4 \mathrm{~h}$ ) in participants without MetS (in blue, A-E) and with MetS (in orange, F-J). Figure S4. Correlation between left atrial (LA) diameter and plasma concentration of negative-charged low-density lipoprotein cholesterol (LDL- $\chi$ ) before (fasting) and after the unified meal (postprandial 0.5, 1, 2, and $4 \mathrm{~h}$ ) in participants without MetS (in blue, A-E) and with MetS (in orange, F-J).

\section{Abbreviations}

AF: Atrial fibrillation; LA: Left atrium; LV: Left ventricle; LVEF: Left ventricle ejection fraction; MetS: Metabolic syndrome; VLDL: Very low density lipoprotein; VLDL-X: Negatively charged very low density lipoprotein

\section{Acknowledgments}

The authors thank Miss Chung-Ya Wang for VLDL sample isolation and purification and Mr. Ming-Chuan Lee for electrocardiography measurements.

\section{Authors' contributions}

All authors have approved the submitted version and agree to be personally accountable for their own contributions. Author Contributions: Conceptualization, Hsiang-Chun Lee; Data curation, Hsiang-Chun Lee; Formal analysis, Hsiang-Chun Lee; Funding acquisition, Hsiang-Chun Lee; Investigation, Hsiang-Chun Lee, Liang-Yin Ke, Shyi-Jang Shin, He-Jyun Jiang and Wei-Chung Tsai; Methodology, Hsiang-Chun Lee and Liang-Yin Ke; Project administration, Hsiang-Chun Lee; Resources, Hsiang-Chun Lee; Software, Hsiang-Chun Lee; Supervision, Hsiang-Chun Lee; Validation, Hsiang-Chun Lee and Liang-Yin Ke; Visualization, Hsiang-Chun Lee; Writing - original draft, Hsiang-Chun Lee; and Writing - review \& editing, Hsiang-Chun Lee.

\section{Funding}

This study was supported by the National Health Research Institutes (NHRIEX107-10724SC and NHRI-EX108-10724SC to H.C. Lee), Kaohsiung Medical University Hospital research grants (KMUH 107-7R11, KMUH 108-8R10); Kaohsiung Medical University (KMU-TP104D04) and Taiwan Ministry of Science and Technology grants (MOST 104-2314-B-037 -080 -MY3 to HCL).

\section{Availability of data and materials}

All the re-identified data are available upon reasonable request (hclee@kmu. edu.tw).

\section{Ethics approval and consent to participate}

Our study was approved by the Kaohsiung Medical University Hospital Institutional Review Board. This study was conducted with adherence to the principles of the Declaration of Helsinki. Signed informed consent was obtained from all subjects prior to engaging in any research.

\section{Consent for publication}

Not applicable.

\section{Competing interests}

"The authors declare no conflict of interest."

\section{Author details}

${ }^{1}$ Center for Lipid Biosciences, Kaohsiung Medical University Hospital, Kaohsiung, Taiwan. 'Lipid Science and Aging Research Center, College of Medicine, Kaohsiung Medical University, Kaohsiung, Taiwan. ${ }^{3}$ Division of Cardiology, Department of Internal Medicine, Kaohsiung Medical University Hospital, Kaohsiung Medical University, Kaohsiung, Taiwan. ${ }^{4}$ Department of Internal Medicine, School of Medicine, College of Medicine, Kaohsiung Medical University, Kaohsiung, Taiwan. ${ }^{5}$ Institute/Center of Medical Science and Technology, National Sun Yat-sen University, Kaohsiung, Taiwan. ${ }^{6}$ School of Medicine, College of Medicine, Kaohsiung Medical University, Kaohsiung, Taiwan. 'Division of Nephrology, Department of Internal Medicine, Kaohsiung Medical University Hospital, Kaohsiung Medical University, Kaohsiung, Taiwan. ${ }^{8}$ Department of Metabolism, Affiliated Hospital of Kaohsiung Medical University, Kaohsiung, Taiwan. ${ }^{9}$ College of Medicine, I-Shou University, Kaohsiung, Taiwan. ${ }^{10}$ Department of Medical Imaging, Kaohsiung Medical University Hospital, Kaohsiung Medical University, Kaohsiung, Taiwan. 
Received: 22 June 2020 Accepted: 10 September 2020 Published online: 22 September 2020

\section{References}

1. Expert Panel on Detection, Evaluation, and Treatment of High Blood Cholesterol in Adults. Executive Summary of The Third Report of The National Cholesterol Education Program (NCEP) Expert Panel on Detection, Evaluation, And Treatment of High Blood Cholesterol In Adults (Adult Treatment Panel III). JAMA. 2001;285(19):2486-97.

2. Korantzopoulos P, Kokkoris S, Papaioannides D. The association of metabolic syndrome with atrial fibrillation: an emerging epidemiological and pathophysiological hypothesis. Cardiology. 2005;104(3):148-9.

3. Tanner RM, Baber U, Carson AP, Voeks J, Brown TM, Soliman EZ, et al. Association of the metabolic syndrome with atrial fibrillation among United States adults (from the REasons for geographic and racial differences in stroke [REGARDS] study). Am J Cardiol. 2011;108(2):227-32.

4. Kim YG, Choi KJ, Han S, Hwang KW, Kwon CH, Park GM, et al. Metabolic syndrome and the risk of new-onset atrial fibrillation in middle-aged east Asian men. Circ J. 2018;82(7):1763-9.

5. Kwon CH, Kim H, Kim SH, Kim BS, Kim HJ, Kim DK, et al. The impact of metabolic syndrome on the incidence of atrial fibrillation: a nationwide longitudinal cohort study in South Korea. J Clin Med. 2019;8(8):1095.

6. Movahed M-R, Hashemzadeh M, Mazen JM. Diabetes mellitus is a strong, independent risk for atrial fibrillation and flutter in addition to other cardiovascular disease. Int J Cardiol. 2005;105(3):315-8.

7. Huxley RR, Filion KB, Konety S, Alonso A. Meta-analysis of cohort and casecontrol studies of type 2 diabetes mellitus and risk of atrial fibrillation. Am J Cardiol. 2011;108(1):56-62.

8. Manolis AJ, Rosei EA, Coca A, Cifkova R, Erdine SE, Kjeldsen S, et al. Hypertension and atrial fibrillation: diagnostic approach, prevention and treatment. Position paper of the working group 'Hypertension arrhythmias and Thrombosis' of the European Society of Hypertension. J Hypertens. 2012:30(2):239-52.

9. Lee HC, Lin YH. The pathogenic role of very low density lipoprotein on atrial remodeling in the metabolic syndrome. Int J Mol Sci. 2020;21(3):891.

10. Toth PP, Fazio S, Wong ND, Hull M, Nichols GA. Risk of cardiovascular events in patients with hypertriglyceridaemia: a review of real-world evidence. Diabetes Obes Metab. 2020;22(3):279-89.

11. Paredes S, Fonseca L, Ribeiro L, Ramos H, Oliveira JC, Palma I. Novel and traditional lipid profiles in metabolic syndrome reveal a high atherogenicity. Sci Rep. 2019:9(1):11792.

12. Sabaka P, Kruzliak P, Gaspar L, Caprnda M, Bendzala M, Balaz D, et al. Postprandial changes of lipoprotein profile: effect of abdominal obesity. Lipids Health Dis. 2013;12(1):179.

13. Mittendorfer B, Yoshino M, Patterson BW, Klein S. VLDL triglyceride kinetics in lean, overweight, and obese men and women. J Clin Endocrinol Metab. 2016;101(11):4151-60.

14. Alves-Bezerra M, Cohen DE. Triglyceride metabolism in the liver. Compr Physiol. 2017;8(1):1-8.

15. Joint committee for guideline. Chinese guidelines for the management of dyslipidemia in adults. J Geriatr Cardiol 2018. 2016;15(1):1-29.

16. Langsted A, Freiberg JJ, Tybjaerg-Hansen A, Schnohr P, Jensen GB, Nordestgaard BG. Nonfasting cholesterol and triglycerides and association with risk of myocardial infarction and total mortality: the Copenhagen City heart study with 31 years of follow-up. J Intern Med. 2011;270(1):65-75.

17. Varbo A, Nordestgaard BG. Nonfasting triglycerides, low-density lipoprotein cholesterol, and heart failure risk: two cohort studies of 113554 individuals. Arterioscler Thromb Vasc Biol. 2018;38(2):464-72.

18. van Ommen B, Keijer J, Kleemann R, Elliott R, Drevon CA, McArdle H, et al. The challenges for molecular nutrition research 2: quantification of the nutritional phenotype. Genes Nutr. 2008;3(2):51-9.

19. Fiamoncini J, Rundle M, Gibbons H, Thomas L, Geillinger-Kästle K, Bunzel D, et al. Plasma metabolome analysis identifies distinct human metabotypes in the postprandial state with different susceptibility to weight loss-mediated metabolic improvements. FASEB J. 2018:0(0):fj.201800330R.

20. Nakajima K, Tanaka A. Atherogenic postprandial remnant lipoproteins; VLDL remnants as a causal factor in atherosclerosis. Clin Chim Acta. 2018;478:200-15.

21. Gallego-Delgado J, Connolly SB, Lazaro A, Sadlier D, Kieran NE, Sugrue DD, et al. Transcriptome of hypertension-induced left ventricular hypertrophy and its regression by antihypertensive therapies. Hypertens Res. 2009;32(5): 347-57.

22. Takahashi S, Sakai J, Fujino T, Hattori H, Zenimaru Y, Suzuki J, et al. The very low-density lipoprotein (VLDL) receptor: characterization and functions as a peripheral lipoprotein receptor. J Atheroscler Thromb. 2004;11(4):200-8.

23. Lee HC, Lin HT, Ke LY, Wei C, Hsiao YL, Chu CS, et al. VLDL from metabolic syndrome individuals enhanced lipid accumulation in atria with association of susceptibility to atrial fibrillation. Int J Mol Sci. 2016;17(1):134.

24. Lee HC, Chen CC, Tsai WC, Lin HT, Shiao YL, Sheu SH, et al. Very-low-density lipoprotein of metabolic syndrome modulates gap junctions and slows cardiac conduction. Sci Rep. 2017;7(1):12050.

25. Shiou YL, Lin HT, Ke LY, Wu BN, Shin SJ, Chen CH, et al. Very low-density lipoproteins of metabolic syndrome modulates STIM1, suppresses storeoperated calcium entry, and deranges myofilament proteins in atrial myocytes. J Clin Med. 2019;8(6):881.

26. Chen CH, Lu J, Chen SH, Huang RY, Yilmaz HR, Dong J, et al. Effects of electronegative VLDL on endothelium damage in metabolic syndrome. Diabetes Care. 2012;35(3):648-53.

27. An K, Zhu J, Ma N, Tang M, Mei J. Predictive risk factors for recurrent atrial fibrillation after modified endoscopic ablation: a 2-year follow-up. Clin Cardiol. 2018:41(3):372-7.

28. Park J, Kim TH, Lee JS, Park JK, Uhm JS, Joung B, et al. Prolonged PR interval predicts clinical recurrence of atrial fibrillation after catheter ablation. J Am Heart Assoc. 2014;3(5):e001277.

29. Nielsen JB, Kühl JT, Pietersen A, Graff C, Lind B, Struijk JJ, et al. P-wave duration and the risk of atrial fibrillation: results from the Copenhagen ECG study. Heart Rhythm. 2015;12(9):1887-95.

30. Magnani JW. Risk assessment for atrial fibrillation: enter the P-wave. Heart Rhythm. 2015;12(9):1896-7.

31. Bancells C, Benitez S, Ordonez-Llanos J, Oorni K, Kovanen PT, Milne RW, et al. Immunochemical analysis of the electronegative LDL subfraction shows that abnormal N-terminal apolipoprotein B conformation is involved in increased binding to proteoglycans. J Biol Chem. 2011;286(2):1125-33.

32. Chan HC, Bonnie Chan HC, Liang CJ, Lee HC, Su H, Lee AS, et al. Role of low-density lipoprotein in early vascular aging associated with systemic lupus Erythematosus. Arthritis Rheumatol. 2020;72(6):972-84.

33. Snyder ML, Soliman EZ, Whitsel EA, Gellert KS, Heiss G. Short-term repeatability of electrocardiographic $P$ wave indices and PR interval. J Electrocardiol. 2014;47(2):257-63.

34. Lang RM, Badano LP, Mor-Avi V, Afilalo J, Armstrong A, Ernande L, et al. Recommendations for cardiac chamber quantification by echocardiography in adults: an update from the American Society of Echocardiography and the European Association of Cardiovascular Imaging. J Am Soc Echocardiogr. 2015;28(1):1-39 e14.

35. Tudorache IF, Trusca VG, Gafencu AV. Apolipoprotein E - a multifunctional protein with implications in various pathologies as a result of its structural features. Comput Struct Biotechnol J. 2017:15:359-65.

36. Niu YG, Evans RD. Very-low-density lipoprotein: complex particles in cardiac energy metabolism. J Lipids. 2011:2011:189876.

37. Feingold KR, Anawalt B, Boyce A, et al. Introduction to Lipids and Lipoproteins. In: Endotext. South Dartmouth (MA): MDText.com, Inc.; 2000

38. Zilversmit DB. Atherogenesis: a postprandial phenomenon. Circulation. 1979; 60(3):473-85.

39. Nordestgaard BG, Langsted A, Mora S, Kolovou G, Baum H, Bruckert E, et al. Fasting is not routinely required for determination of a lipid profile: clinical and laboratory implications including flagging at desirable concentration cut-points-a joint consensus statement from the European atherosclerosis society and European Federation of Clinical Chemistry and Laboratory Medicine. Eur Heart J. 2016;37(25):1944-58.

40. Nakajima K, Tokita Y, Tanaka A, Takahashi S. The VLDL receptor plays a key role in the metabolism of postprandial remnant lipoproteins. Clin Chim Acta. 2019:495:382-93.

41. Wang TJ, Parise H, Levy D, D'Agostino RB Sr, Wolf PA, Vasan RS, et al. Obesity and the risk of new-onset atrial fibrillation. JAMA. 2004;292(20): 2471-7.

42. Nystrom PK, Carlsson AC, Leander K, de Faire U, Hellenius ML, Gigante B. Obesity, metabolic syndrome and risk of atrial fibrillation: a Swedish, prospective cohort study. PLoS One. 2015:10(5):e0127111.

43. Mahajan R, Lau DH, Brooks AG, Shipp NJ, Manavis J, Wood JP, et al. Electrophysiological, Electroanatomical, and structural remodeling of the atria as consequences of sustained obesity. J Am Coll Cardiol. 2015;66(1):1-11. 
44. Kim SM, Cho Kl, Kwon JH, Lee HG, Kim TI. Impact of obstructive sleep apnea on left atrial functional and structural remodeling beyond obesity. J Cardiol. 2012;60(6):475-83.

45. Choisy SC, Arberry LA, Hancox JC, James AF. Increased susceptibility to atrial tachyarrhythmia in spontaneously hypertensive rat hearts. Hypertension. 2007;49(3):498-505.

\section{Publisher's Note}

Springer Nature remains neutral with regard to jurisdictional claims in published maps and institutional affiliations.

- fast, convenient online submission

- thorough peer review by experienced researchers in your field

- rapid publication on acceptance

- support for research data, including large and complex data types

- gold Open Access which fosters wider collaboration and increased citations

- maximum visibility for your research: over $100 \mathrm{M}$ website views per year

At $\mathrm{BMC}$, research is always in progress. 